(C)2007 IEEE. Personal use of this material is permitted. However, permission to reprint/republish this material for advertising or promotional purposes or for creating new collective works for resale or redistribution to servers or lists, or to reuse any copyrighted component of this work in other works must be obtained from the IEEE 


\title{
Ascertaining Risk in Financial Terms in Digital Business Ecosystem Environments
}

\author{
Omar Khadeer Hussain, Elizabeth Chang, Farookh Khadeer Hussain and Tharam S. Dillon \\ Digital Ecosystems and Business Intelligence Institute, Curtin University of Technology, Perth, Australia \\ e-mail: Omar.Hussain@postgrad.curtin.edu.au, \{Elizabeth.Chang, Farookh.Hussain, Tharam.Dillon\}@cbs.curtin.edu.au
}

\begin{abstract}
In a digital business ecosystem environment the trusting agent by analysing beforehand the possible risk in interacting with a probable trusted agent, can make a better decision of its future course of interaction with it. A possible outcome of Risk is the loss to trusting agent's resources involved in the interaction. In a financial interaction, the possible loss that may be incurred is usually the monetary loss in the resources of the trusting agent that are involved in the interaction. In this paper, we propose a methodology by which the trusting agent can determine beforehand the possible Risk in financial terms or the possible loss to its resources as a result of interacting with a probable trusted agent.
\end{abstract}

Index Terms - FailureLevel, Risk, Time slot, Trusting agent, Trusted agent.

\section{INTRODUCTION}

The significance for the trusting agent to analyse the possible risk in interacting with a probable trusted agent to achieve its desired outcomes is substantial. The trusting agent, by analysing the possible risk beforehand, could determine whether it will achieve its desired outcomes from the interaction or not and also gain an idea or direction in which its interaction might head. Risk is important in the study of behaviour in e-commerce as there is a whole body of literature based in rational economics that argues that the decision to buy is based on the risk-adjusted cost-benefit analysis [1]. Risk plays a central role in deciding whether to proceed with a transaction or not. It can broadly be defined as an attribute of decision making that reflects the variance of its possible outcomes. Thus, it commands a central role in any discussion that is related to a transaction.

Digital Business Ecosystems is a new concept that is emerging worldwide as an innovative approach to support the adoption and development of information and communication technologies. Digital ecosystems transcend the traditional, rigorously defined, collaborative environments from centralised or distributed or hybrid models into an open, flexible, domain clustered and demand-driven interactive environment. A digital ecosystem is a new-networked architecture and collaborative environment that addresses the weakness of client-server, peer-to-peer, grid and web services. It is a self-organising digital infrastructure aimed at creating a digital environment for networked organisations that supports the cooperation, knowledge sharing, development of open and adaptive technologies and the evolutionary business models [2-4]. It can also be defined as a system which is loosely coupled, agent-based collaborative environment where every species is proactive and responsive and acts for its own benefit or profit. A business ecosystem is the network of buyers, suppliers and makers of related products or services plus the socio-economic environment. An agent in a Digital Ecosystem can be a client and a server at the same time. They may offer their service to others as a server and request help as a client. The communication and collaboration is through swarm intelligence. Unlike traditional environments, digital ecosystems are self-organising systems which can form different architectural models through swarm intelligence. A demand driven business ecosystem interaction implies that the trusting agent wants to achieve certain desired outcomes in its future interaction and, based on that, it selects a trusted agent to interact with, who can fulfil its demand. In doing so, it is possible that the trusting agent has to determine which trusted agent to interact with from a set of probable trusted agents. The trusting agent can ease the decision making process of which agent to interact with, or it can firm its decision whether to interact with a trusted agent or not by analysing the possible risk beforehand in interacting with them according to its demand. The possible risk in an interaction is a combination of:

- The probability of failure in achieving the outcome; and

- The possible consequences of failure.

The trusting agent has to determine these two aspects to analyse and quantify the possible risk in an interaction. In this paper, we propose a methodology by which the trusting agent can determine the possible risk in financial terms in interacting with a probable trusted agent according to the demand of its interaction. We will propose and explain the methodology in the next sections. This paper is organised into six sections. In section 2, we discuss our previous work for ascertaining the FailureLevel in an interaction. In section 3 and 4 , we propose the methodology of determining the possible consequences of failure in an interaction or the possible risk in financial terms in an interaction. In section 5, we explain the proposed methodology with an example and in section 6 we conclude the paper.

\section{RELATED WORK}

As mentioned earlier, the trusting agent has to determine the probability of failure of the interaction and the possible consequences of failure to its resources in order to analyse the possible level of Risk before initiating its interaction with a trusted agent. To quantify and represent semantically the probability of failure of an interaction, we defined the term 'FailureLevel' and the Failure scale in Hussain et al. [5]. FailureLevel quantifies and expresses semantically the 
possible level of failure in the interaction on the failure scale. The Failure scale as shown in Figure 1 represents the different possible levels of failure that could be possible in an interaction. The trusting agent determines the FailureLevel in interacting with the probable trusted agent beforehand by ascertaining its in-capability to complete the interaction, according to the expectations of its future interaction with it. In other words, the FailureLevel of an interaction is the extent to which the trusting agent thinks that it might not achieve its desired outcomes in interacting with a probable trusted agent.

\begin{tabular}{|c|c|c|c|}
\hline $\begin{array}{l}\text { Semantics of } \\
\text { Failure Level }\end{array}$ & $\begin{array}{l}\text { Probability of } \\
\text { Failure }\end{array}$ & FailureLevels & Star Rating \\
\hline Unknown & & -1 & Not Displayed \\
\hline Total Failure & $\begin{array}{c}91-100 \% \text { Probability of } \\
\text { Failure }\end{array}$ & 0 & Not Displayed \\
\hline Extremely High & $\begin{array}{c}71-90 \% \text { Probability of } \\
\text { Failure }\end{array}$ & 1 & From \\
\hline Largely High & $\begin{array}{c}51-70 \% \text { Probability of } \\
\text { Failure }\end{array}$ & 2 & 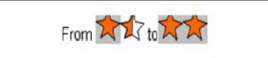 \\
\hline High & $\begin{array}{c}26-50 \% \text { Probability of } \\
\text { Failure }\end{array}$ & 3 & 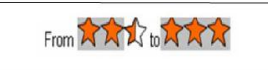 \\
\hline Significantly Low & $\begin{array}{l}11-25 \% \text { Probability of } \\
\text { Failure } \\
\end{array}$ & 4 & From WWWLtoWWW \\
\hline Extremely Low & $\begin{array}{c}0-10 \% \text { Probability of } \\
\text { Failure }\end{array}$ & 5 & 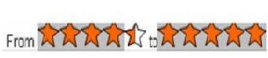 \\
\hline
\end{tabular}

The expectations or the desired outcomes that the trusting agent wants in its interaction with a probable trusted agent can be termed at a higher level as the 'context' of the interaction. In other terms context represents the high level nature of the trusting agent's interaction with the probable trusted agent [7]. It can be decomposed into several detailed aspects known as the criteria. Criteria is defined as the demand or the set of factors which show specifically what the trusting agent wants in its interaction with the trusted agent in the particular context. By considering the expectations of its future interaction, the trusting agent will accurately determine the probability of failure in its interaction according to its criteria.

The possible interaction of the trusting agent with the probable trusted agent is in the future state of time. Hence for risk analysis, the trusting agent has to determine the FailureLevel in interacting with the probable trusted agent in that future state of time. In order to achieve that, we propose that the trusting agent analyse the FailureLevel in interacting with a probable trusted agent in two stages. They are:

1. Pre-interaction start time phase

2. Post-interaction start time phase

Pre-Interaction start time phase refers to the period of time before the trusting agent starts its interaction with the probable trusted agent, whereas Post-Interaction start time phase is that period of time after the trusting agent starts and interacts with the probable trusted agent. For risk analysis, the trusting agent has to determine the FailureLevel in interacting with a probable trusted agent in this period of time, that is in the post-interaction start time phase. However, as this time phase is in the future state of time, the trusting agent can only determine it by using some prediction methods. So to achieve this we propose that the trusting agent should first ascertain the FailureLevel of the probable trusted agent according to the specific context and criteria as that of its future interaction in the pre-interaction start time phase. Based on those levels the trusting agent can determine or predict it's FailureLevel in the postinteraction start time phase. The determined FailureLevel of the probable trusted agent in that time phase depicts the probability of failure in interacting with it, during the time of the trusting agent's interaction with it.

As mentioned in the literature, Risk is dynamic - varying from time to time. Hence, the trusting agent should take this dynamic nature of risk into consideration while doing risk analysis. To incorporate that we propose the trusting agent should divide the total time that it considers to determine the FailureLevel of the probable trusted agent, termed as the 'time space', into different non-overlapping parts, termed as the 'time slots', and determine the FailureLevel of the trusted agent in each of those time slots. By doing so the trusting agent ascertains the correct FailureLevel of the probable trusted agent in a time slot, according to its incapability to complete the criterions of its future interaction in that particular time slot, thus considering its dynamic nature while doing risk analysis. The time slots will be spread out either in the pre-interaction or in the post-interaction start time phase. The trusting agent has to determine the FailureLevel of the probable trusted agent in each time slot according to the time phase in which they fall. The methodology for determining the FailureLevel of the probable trusted agent in both the pre- and postinteraction start time phase is defined in Hussain et al [5]. In this paper, due to space limitation we will give only a brief overview of the methodology.

In the methodology we propose that the trusting agent in each of the pre-interaction phase time slots determines a crisp FailureLevel of the probable trusted agent on the Failure scale either by considering its previous interaction history with it or by soliciting for recommendations and then assimilating them according to the criteria of its future interaction. Further while assimilating the recommendations the trusting agent gives more weight to those recommendations which are from trustworthy recommending agents as compared to those from the unknown recommending agents. The recommendations from unknown recommending agents are omitted and not considered by the trusting agent. To reflect the 'fresh' nature of the probable trusted agent while assimilating the recommendations we propose that the trusting agent gives more weight to those recommendations which are in the recent time slot to the time spot of its interaction with a probable trusted agent, as compared to those which are in the far recent ones.

After determining the FailureLevel of the probable trusted agent in each of the pre-interaction start time slots, the trusting agent can utilize those to predict or ascertain its FailureLevel in the post-interaction start time phase. We propose that the trusting agent while determining the FailureLevel of a probable trusted agent in a postinteraction start time slot, should determine the magnitude of occurrence of each level of failure within the domain of 
$(0,5)$ on the Failure scale rather than determining a crisp FailureLevel for that time slot as it does in the PreInteraction start time phase. This is due to the fact that the FailureLevel of a probable trusted agent at time ' $t+1$ ' is predicted by considering its FailureLevels from its time space till time ' $t$ '. The prediction might not give an accurate conclusion as compared to the one obtained in the pre-interaction start time phase where the trusting agent determines the FailureLevel of a probable trusted agent by considering concrete data i.e. either its past interaction history with the trusted agent or by soliciting and assimilating recommendations from other agents. To overcome that, we propose that the trusting agent instead of concluding with a crisp FailureLevel of the trusted agent in each of the post-interaction start time slots, should determine the magnitude of presence of each level of failure on the Failure scale; within the domain of $(0,5)$. Doing so would also give the trusting agent an indication of the probability of occurrence of each level of failure present in interacting with the probable trusted agent in a particular time slot. Further, by representing the FailureLevel in interacting with the probable trusted agent in each time slot of the post-interaction start time phase by busbars of the different possible levels of failure; the trusting agent would get a better indication of how the probable trusted agent might behave in the interaction. As the FailureLevel of a probable trusted agent in the pre-interaction start time phase is determined strictly according to the criteria of its future interaction, the future FailureLevel (FFL) determined by utilising these levels too is strictly according those criteria.

For better understanding, let us consider an example of trusting agent ' $A$ ' wanting to interact with a trusted agent ' $B$ ' in context ' $C$ '. The criteria that it wants in the interaction are $\mathrm{C} 1$ and $\mathrm{C} 2$. The trusting agent ' $\mathrm{A}$ ' does not have any past interaction with the trusted agent and in order to analyse the Risk before initiating the interaction, it solicits for recommendations to determine the possible FailureLevel in the interaction. Let us suppose that the trusting agent divides the time space into six equal time slots with four in the pre-interaction time phase ( $\mathrm{t}-4$ till $\mathrm{t}-1)$ and two in the post-interaction start time phase ( $t 1$ and $\mathbf{t} 2$ ). From the recommendations achieved, the trusting agent classifies them according to time, trustworthiness and assimilates them according to the criteria of its future interaction by using the methodology defined in Hussain et al. [5] to determine a crisp FailureLevel of the trusted agent in each of the pre-interaction start time slots. Based on the determined FailureLevel of the trusted agent in the preinteraction time slots the trusting agent ascertains the FailureLevel of the trusted agent in the post-interaction time phase by using the methodology defined in Hussain et al. [5]. The determined FailureLevel of the trusted agent in the post-interaction time phase is strictly according the criteria of its future interaction with it. As mentioned earlier, in the post-interaction time slots the trusting agent determines the probability of occurrence of each level of failure on the Failure scale. Let us suppose the FailureLevel determined for the trusted agent in the post-interaction time slots ( $\mathrm{t} 1$ and $\mathrm{t} 2$ ) are as shown in figure 2 and 3 respectively.

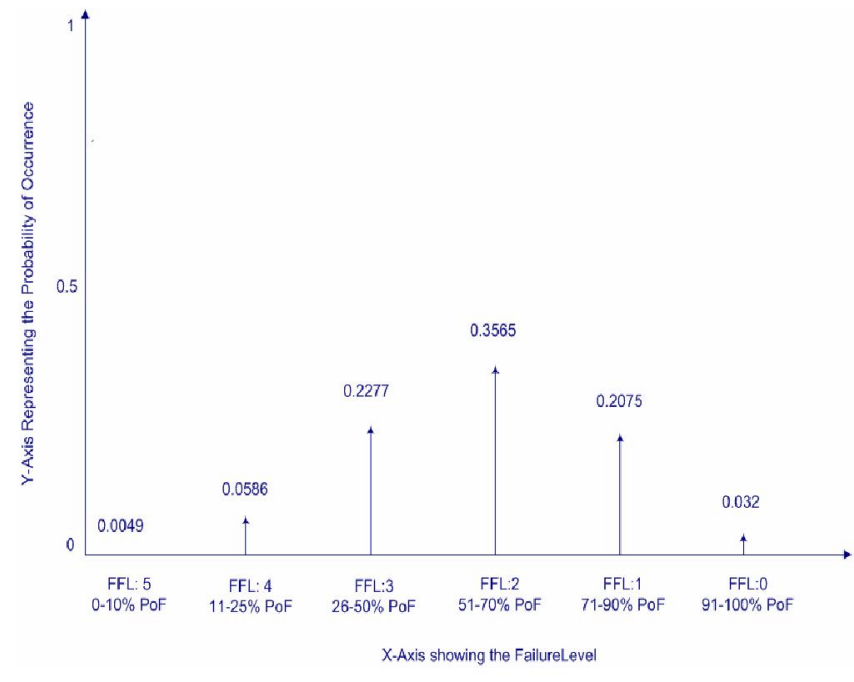

Figure 2: Magnitude of occurrence of each level of failure in time slot $\mathrm{t} 1$

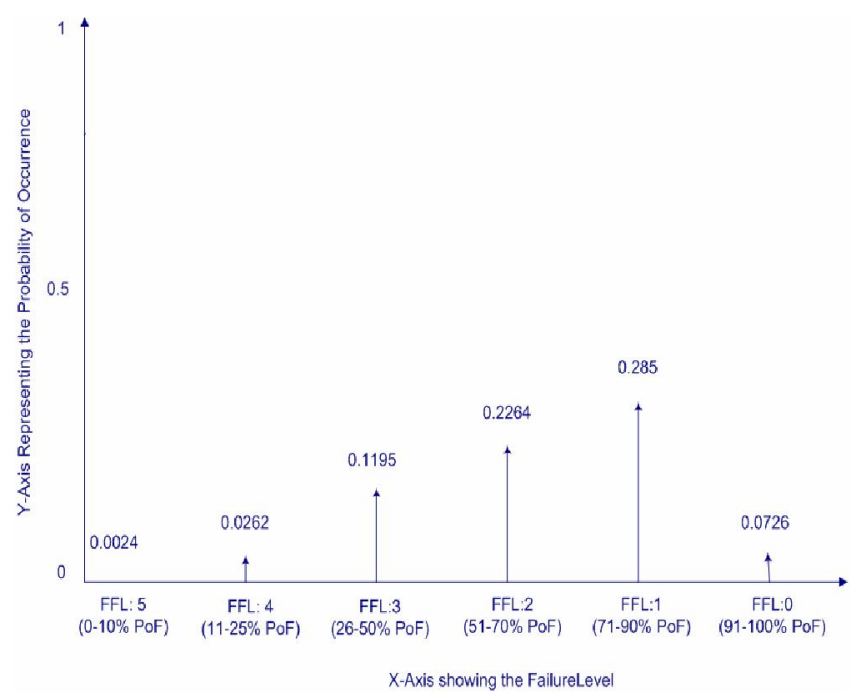

Figure 3: Magnitude of occurrence of each level of failure in time slot $t 2$

Once the FailureLevel in interacting with a probable trusted agent has been determined, the trusting agent should then determine the possible consequences of failure to its resources to ascertain the possible Risk in the interaction. In the next sections, we propose a methodology by which the trusting agent can determine the possible consequences of failure in an interaction.

\section{DETERMINING THE AMOUNT INVESTED CURVE}

The possible consequences of failure in an interaction are usually to the resources of the trusting agent that are involved in it. In a digital business ecosystem environment a trusting agent interacts with a trusted agent according to the demand of its interaction. Hence, the resources invested by the trusting agent in the interaction might vary according to its demand. It can vary from being anything like the mutual exchange of goods between the agents or the receipt of certain products by the trusting agent from the trusted agent in exchange of the monetary value. In this paper, we assume that the trusting agent interacts with the probable trusted agent to achieve its demand in exchange of the 
monetary financial value. Subsequently, in our context, the term 'resources' refers to the financial resources invested by the trusting agent in its interaction with the trusted agent to achieve its demand. By saying 'the trusting agent has to determine the consequences of failure to its resources in an interaction', we mean the trusting agent has to determine the possible consequences of failure to its financial resources involved in the interaction.

In contrast to determining the FailureLevel of the interaction, the consequences of failure are determined only in the post-interaction start time phase as this is the time in which the trusting agent interacts with a probable trusted agent and has its resources at stake. As the FailureLevel of an interaction is determined in each time slot of the postinteraction time phase, the possible consequences of failure too should be determined in those to ascertain the possible Risk in each of the post-interaction time slots.

The number of time slots in the post-interaction phase depends on the trusting agent's classification of the time space of the interaction. It may be the case that there is only one or more than one time slots in the post-interaction start time phase. In order to determine the possible consequences of failure to its resources in a time slot, the trusting agent has to first determine the range and threshold of its resources that it has at stake in that time slot. If there is more than one time slot in the post-interaction phase then the net worth of the trusting agent's resources at stake in the interaction increases progressively as the time slots increase according to the total worth invested in each of them. To explain with an example, let us consider our previous discussion of the trusting agent ' $\mathrm{A}$ ' wanting to interact with a trusted agent ' $\mathrm{B}$ '. In its interaction there are two time slots in the post-interaction time phase. In the first time slot the trusting agent invests $\$ 15,000$ in the interaction, hence, the maximum threshold of its resources at stake and the total worth of the interaction at the end of the first time slot is $\$ 15,000$. In the second time slot, it invests $\$ 5,000$ in the interaction and subsequently the net worth of its resources at stake is $\$ 20,000$ at the end of the second time slot. But at the beginning of the second time slot, the net worth of the interaction is already $\$ 15,000$ which is the total amount invested in the first time slot. While determining the possible consequences of failure to its resources in the second time slot of its interaction, the trusting agent should consider the fact that the range of its net resources at stake in that time slot is from $\$ 15,000$ to $\$ 20,000$, and not from $\$ 0$ to $\$ 5,000$, as this is the range of amount invested in that particular time slot.

Moreover it is possible that in a time slot, the trusting agent may invest its resources in a stepwise way rather than investing the maximum threshold at once. For example, let us consider that the trusting agent divides its time space in such a way so that the duration of each time slot is 7 days. During the first time slot of the post-interaction phase, it is possible that the trusting agent ' $A$ ' may invest the total worth of the time slot i.e. $\$ 15,000$ in the beginning or it may invest its resources in a stepwise way, that is in the order of $\$ 2,000 ; \$ 3,000 ; \$ 10,000$ on days 1,3 and 6 of the time slots respectively to gradually make the total worth of the time slot $\$ 15,000$. Subsequently when the trusting agent determines the possible consequences of failure to its resources in that time slot, it should consider the way the resources were invested in the interaction. This is because when the trusting agent invests $\$ 15,000$ at the beginning of the time slot, the total amount of its resource that it has at stake throughout the time slot is $\$ 15,000$. The possible consequences of failure in its resources have to be determined by considering that worth during the time slot of 7 days. On the other hand, if the resources were invested in a stepwise way as explained earlier then the total worth of its resources at stake reaches $\$ 15,000$ on day 6 of the time slot. This means that the trusting agent has $\$ 15,000$ worth of resources at stake for only 2 days out of the 7 day period of the time slot. So when determining the possible consequences of failure to its resources in a time slot, the trusting agent should do it according to the accurate net worth of its resources that it has at stake throughout that time slot. Hence, the trusting agent should first ascertain the probability of the net worth of its resources at stake throughout the time slot and then determine the subsequent possible consequences of failure to it accordingly. A point to be noted here is that when the resources are invested in a stepwise way and when the total worth of the interaction becomes $\$ 5,000$ on day 3 of the time slot, then the amount invested $(\$ 2,000)$ during days 1 to 3 of the time slot also have to be considered as it counts towards the total worth of the interaction.

To achieve that, we propose the calculation of an Amount Invested Curve. This curve gives the probability of an amount at stake throughout the duration of the time slot. Another important property of this probabilistic model of the amount invested curve is that it describes the probability of the worth of the interaction to be at least a certain amount throughout the duration of the time slot. To calculate the amount invested curve we utilise the Fundamental Probability Formulae to determine the probability of an amount being invested throughout the time slot.

Let us represent an amount invested throughout the time slot by the discrete random variable ' $\mathrm{X}$ '. We determine the probability mass function (PMF) of $X$ by using the probability theory and then utilise the fundamental probability formulae to determine the amount invested curve by finding the probability of the discrete random variable ' $\mathrm{X}$ ' being present throughout the duration of the time slot. By determining the probability for its total worth which is at stake in a particular time slot, the trusting agent can determine accurately the possible consequences of failure to it.

To obtain the amount invested curve for our example, let us consider that the trusting agent invests $\$ 20,000$ in the interaction. Out of that, $\$ 15,000$ is invested in the first time slot and $\$ 5,000$ in the second time slot to make the total worth of the interaction $\$ 20,000$. Further let us consider that the trusting agent invests its resources in a step wise fashion in both the time slots $\mathrm{t} 1$ and $\mathrm{t} 2$. It invests $\$ 2,000$; $\$ 3,000 ; \$ 6,000$ and $\$ 4,000$ on days $1,3,4$ and 6 respectively of the first time slot to gradually make the total worth of the first time slot $\$ 15,000$ and $\$ 2,000 ; \$ 2,000$ and 
$\$ 1,000$ on days 2,3 and 6 respectively of the second time slot to make the total worth of the interaction $\$ 20,000$. Determining and representing in Figures 4 and 5 respectively the amount invested curve for the time slots $\mathrm{t} 1$ and $\mathrm{t} 2$ by using the probability function formulae.

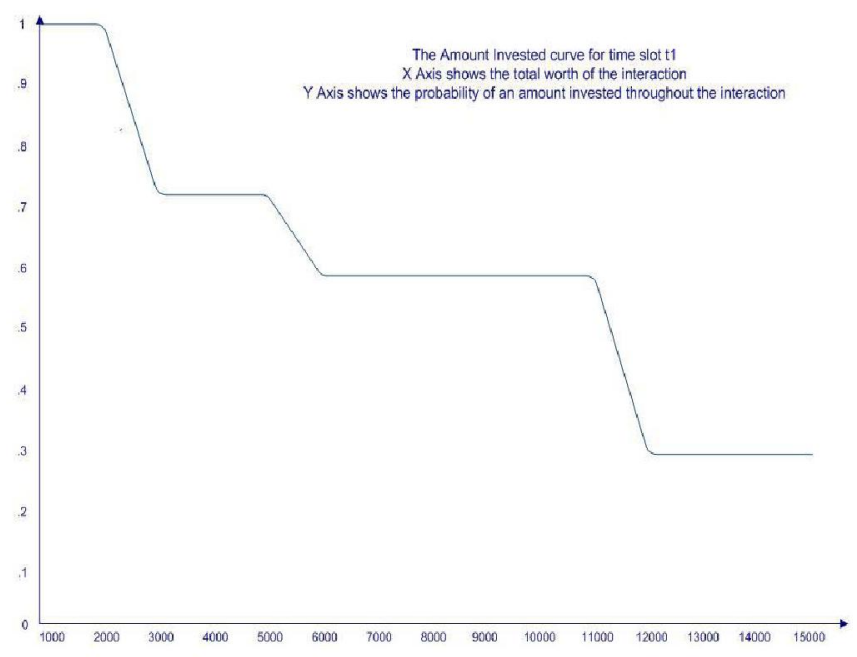

Figure 4: Amount invested curve for time slot $\mathrm{t} 1$

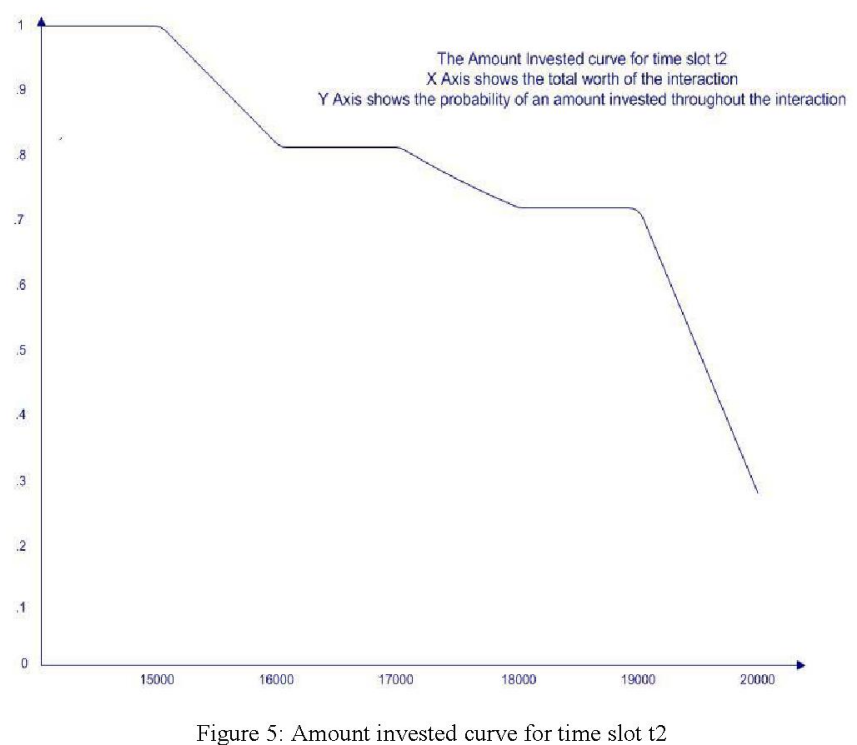

By the nature of the trusting agent investing its resources in time slot $\mathbf{t} 1$, it can be seen from the amount invested curve of figure 4 that the probability of the trusting agent's resources of worth $\$ 1,000$ at stake throughout the time slot $\mathrm{t} 1$ is $100 \%$ where as the probability of the resources of worth $\$ 15,000$ at stake throughout the time slot $\mathrm{tl}$ is $28.57 \%$. Hence, when determining the possible consequences of failure to its resources in that time slot, the trusting agent should ascertain it according to the accurate worth that it has at stake throughout that time slot.

The essence of the amount invested curve is that it gives the probability of an amount at stake throughout the duration of the time slot from the trusting agent's total worth of resources invested. The importance of determining such a curve is to ascertain the consequences of failure in a time slot accurately according to worth of the trusting agent's resources at stake as explained earlier.

In the next section, we will propose the methodology by which the trusting agent can determine the possible Risk in financial terms in each time slot of its interaction.

\section{DETERMINING THE LOSS CURVE OF A TIME SLOT}

Once the trusting agent ascertains the FailureLevel of the trusted agent and the amount invested curve for each time slot of the post-interaction phase, it can then ascertain the possible consequences of failure or the possible Risk in financial terms to its resources in each of those time slot. We represent the FailureLevel and the amount invested curve of each time slot in the post interaction phase as a probability function. They are then combined by using a mathematical operation known as 'convolution' to determine the 'loss curve' which shows the possible consequences of failure or the possible Risk in financial terms in each time slot of the post-interaction phase.

Convolution is an operator that takes two input functions $f$ and $g$ and produces an output function $h$. In other terms, convolution is an integral that expresses the amount of overlap of one function $f$ as it is shifted over another function $g$ [6]. The output function $h$, showing the loss curve, is the result of overlap between the two inputs namely the FailureLevel of the trusted agent, represented by function $f$ and the amount invested curve represented by function $g$. Convolution is represented by the symbol $\otimes$. Hence,

\section{Possible Risk in Financial Terms $=$ FailureLevel $\otimes$ Amount Invested}

The convolution of two functions $f$ and $g$ over an infinite range is determined as:

$$
\mathrm{f} \otimes \mathrm{g} \Rightarrow h=\int_{-\infty}^{\infty} f(\tau) g(t-\tau) d \tau
$$

Alternately the convolution of two functions can also be written as:

$$
\mathrm{f} \otimes \mathrm{g} \Rightarrow h=\int_{-\infty}^{\infty} \mathrm{g}(\tau) f(t-\tau) d \tau
$$

By using the above approach, the trusting agent can determine the loss curve for each time slot of the postinteraction phase. The achieved curve gives the probability of the trusting agent loosing an amount from its invested resources in a time slot by considering the FailureLevel of the trusted agent in that time slot and the probability of that amount being at stake throughout the duration of that time slot. The concept will be explained further in the next section when we demonstrate it by using an example.

\section{EXAMPLE OF ASCERT AINING THE POSSIBLE RISK IN FINANCIAL TERMS IN AN INTERACTION}

In this section, we will demonstrate with an example the proposed methodology of ascertaining the loss curve of each time slot. Let us consider the scenario of an interaction discussed in the previous sections of a trusting agent ' $A$ ' 
wanting to interact with the probable trusted agent ' $\mathrm{B}$ ' in context ' $\mathrm{C}$ '. Before initiating the interaction, the trusting agent decides to analyse the possible Risk present in interacting with the trusted agent according to its demand or criteria. As mentioned earlier, there are two time slots in the post-interaction time phase and the trusting agent has to analyse the possible Risk in those.

To analyse the possible Risk to its resources in the interaction, the trusting agent has to determine the FailureLevel of the trusted agent and the amount invested curve for each time slot and combine them by using the convolution operator to determine the loss curve.

The FailureLevel of the trusted agent in time slots $\mathbf{t} 1$ and $\mathrm{t} 2$ in the post-interaction phase are represented in Figures 2 and 3 respectively and the amount invested curve for those time slots as represented in Figure 4 and 5 respectively. Combining these two aspects of risk by using the convolution operator to determine the loss curve for each time slot of the post-interaction time phase and representing it in Figures 6 and 7 respectively we get;

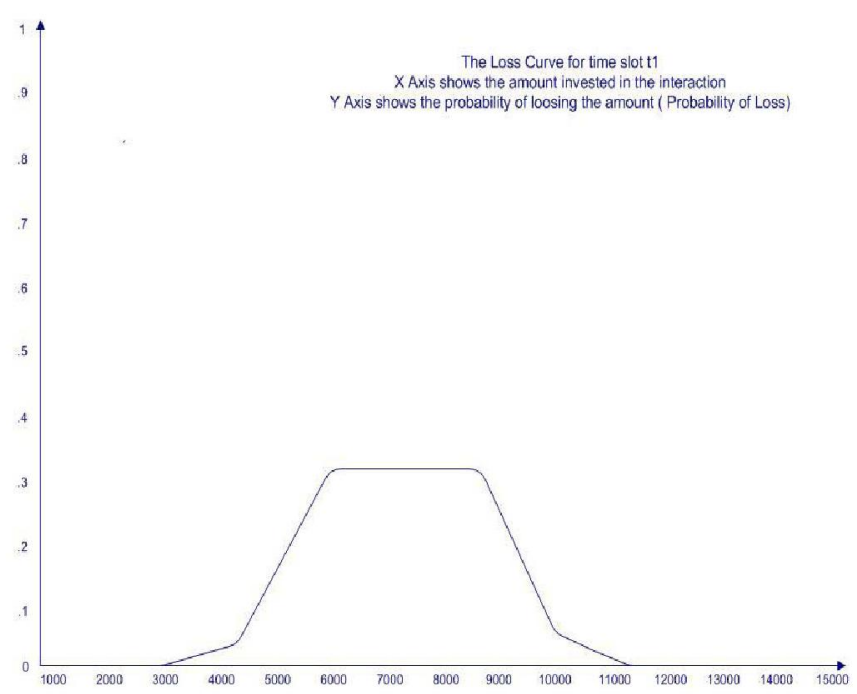

Figure 6: Quantifying the possible Risk in financial terms in time slot $\mathrm{t} 1$

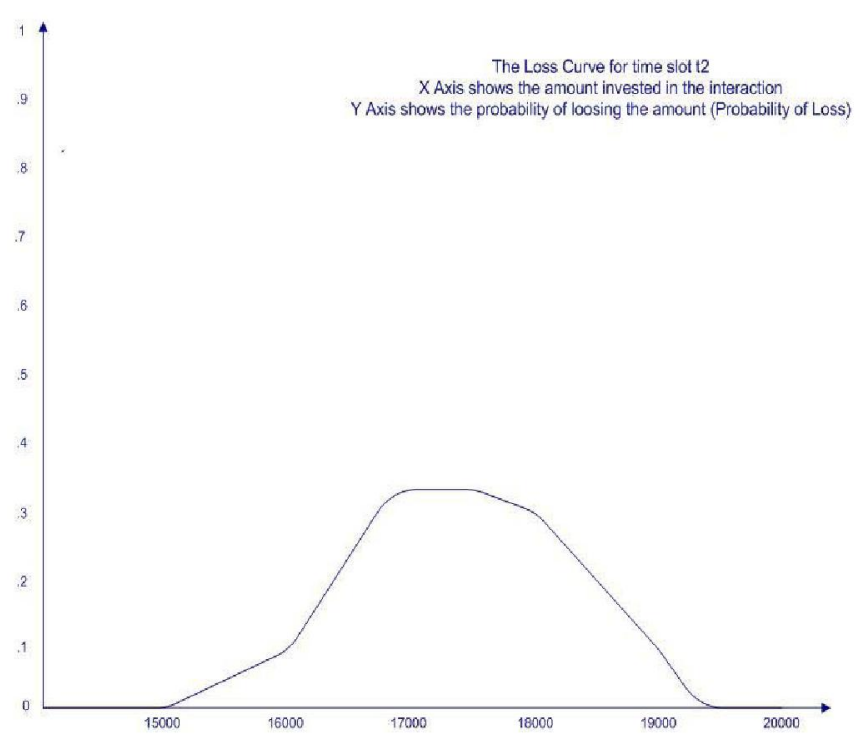

Figure 7: Quantifying the possible Risk in financial terms in time slot $\mathrm{t} 2$
The loss curve of each time slot represents the possible consequences of failure to the trusting agent's resources in interacting with the trusted agent, in that time slot of the post-interaction phase. From Figure 6, it can be seen that in time slot tl the probability of the trusting agent loosing $\$ 6,000$ from its total resources invested of $\$ 15,000$ is $33 \%$. The loss curve of a time slot quantifies in financial terms the possible risk to the trusting agent's resources in that time slot. The trusting agent can make an informed decision of its future course of action with a probable trusted agent by analysing the possible level of Risk in interacting with it. Also, by using the proposed approach, it can choose an agent to interact with from a set of probable trusted agents after analysing the possible Risk in interacting with each of them.

\section{CONCLUSION}

In this paper, we highlighted an important characteristic of determining the possible Risk in an interaction, namely its impact in financial terms on the trusting agent's resources. We proposed an approach by which the trusting agent can determine beforehand the possible Risk to its resources in interacting with a probable trusted agent in a digital business ecosystem domain. We make use of the mathematical operator convolution in our approach. The possible Risk in an interaction is determined by convolving the probability of failure in interacting with the trusted agent with the level of resources that the trusting agent invests and has at stake in the interaction. Once the trusting agent gets an idea of the possible financial loss to its resources in interacting with a probable trusted agent, then it can make a more informed decision of its future course of action with it or it can choose an agent to interact with among the set of probable trusted agents.

\section{REFERENCES}

[1] S. Greenland, 'Bounding analysis as an inadequately specified methodology', Risk Analysis vol. 24, no. 5, pp. 1085-1092, 2004.

[2] Technologies for Digital Ecosystems, Available: http://www.digitalecosystems.org/, Retrieved on 6 October 2006.

[3] G.I. Doukidis, N. Mylonopoulos and N. Pouloudi, 'Social and economic transformation in the digital era', Idea Group International, ISBN: 1591402670,2003

[4] P.J. Denning, R.M. Metcalfe and M. Robert, 'Beyond calculation: The next fifty years of computing', (edited) Springer ISBN: $0387985883,1998$.

[5] O.K. Hussain, E. Chang, F.K. Hussain and T.S. Dillon, "A methodology to quantify failure for risk-based decision support system in Digital Business Ecosystems', Accepted for publication in Journal of Data and Knowledge Engineering (DKE), Elsevier Science.

[6] E.W. Weisstein, 'Convolution.' From MathWorld--A Wolfram Web Resource. http://mathworld.wolfram.com/Convolution.html, Retrieved on 30 December 2006.

[7] E. Chang, T. Dillon, F. K. Hussain, 'Trust and Reputation for Service-Oriented Environments: Technologies for Building Business Intelligence and Consumer Confidence', 1st edition, John Wiley and Sons Ltd, ISBN: 0-470-01547-0, 2006. 